\title{
Clustering and relaxation in Hamiltonian long-range dynamics
}

\author{
Mickael Antoni ${ }^{1}$ and Stefano Ruffo ${ }^{2, *}$ \\ ${ }^{1}$ Istituto Nazionale di Fisica della Materia, Laboratorio FORUM, Largo Enrico Fermi 2, 50125 Firenze, Italy \\ ${ }^{2}$ Laboratoire de Physique Quantique, Université Paul Sabatier, CNRS, URA 505, Toulouse, France
}

(Received 15 February 1995)

\begin{abstract}
We study the dynamics of a fully coupled network of $N$ classical rotators, which can also be viewed as a mean-field $X Y$ Heisenberg (HMF) model, in the attractive (ferromagnetic) and repulsive (antiferromagnetic) cases. The exact free energy and the spectral properties of a Vlasov-Poisson equation give hints on the values of dynamical observables and on time relaxation properties. At high energy (high temperature $T$ ) the system relaxes to Maxwellian equilibrium with vanishing magnetization, but the relaxation time to the equilibrium momentum distribution diverges with $N$ as $N T^{2}$ in the ferromagnetic case and as $N T^{3 / 2}$ in the antiferromagnetic case. The $N$ dependence of the relaxation time is suggested by an analogy of the HMF model with gravitational and charged sheets dynamics in one dimension, and is verified in numerical simulations. Below the critical temperature the ferromagnetic HMF model shows a collective phenomenon where the rotators form a drifting cluster; we argue that the drifting speed vanishes as $N^{-1 / 2}$ but increases as one approaches the critical point (a manifestation of critical slowing down). For the antiferromagnetic HMF model a two-cluster drifting state with zero magnetization forms spontaneously at very small temperatures; at larger temperatures an initial density modulation produces this state, which relaxes very slowly. This suggests the possibility of exciting magnetized states in a mean-field antiferromagnetic system.
\end{abstract}

PACS number(s): 05.45.+b, 05.70.Fh, 05.20.Dd

\section{INTRODUCTION}

In this paper we study in detail a simple model of $N$ interacting particles moving on the unit circle [1]. The coordinate $-\pi<\theta_{i} \leq \pi$ of particle $i$ is its position on the circle and $p_{i}$ its conjugate momentum. The equations of motion derive from the following Hamiltonian:

$$
H=\sum_{i=1}^{N} \frac{p_{i}^{2}}{2}+\frac{\epsilon}{2 N} \sum_{i, j=1}^{N}\left[1-\cos \left(\theta_{i}-\theta_{j}\right)\right]=K+V,
$$

where $K$ and $V$ are the kinetic and the potential energy, respectively. Each particle interacts with all others and thus moves in a force field which is, at each time, the sum of the individual fields produced by all the others. The interaction strength is rescaled by the number of particles, making the potential thermodynamically stable [2]. Defining a spin vector associated to each particle

$$
\mathbf{m}_{i}=\left(\cos \theta_{i}, \sin \theta_{i}\right),
$$

we observe that the interaction term in (1) corresponds to the Heisenberg $X-Y$ interaction, in the infinite range mean-field case. Therefore, we call this model Hamiltonian the mean-field $X-Y$ model (HMF). The positive interaction case, $\epsilon>0$, corresponds to the ferromagnetic model; the $\epsilon<0$ case corresponds to the antiferromagnetic one. The thermodynamics of model (1) can be derived exactly (see Sec. II), but its nonequilibrium dynam-

\footnotetext{
*Permanent address: Dipartimento di Energetica, Università di Firenze, Via S. Marta 3, 50139 Firenze, Italy and INFN, Sezione di Firenze and INFM, Unità di Firenze, Firenze, Italy.
}

ical behavior cannot (see Secs. III and IV). This shows up clearly if we write down the equations of motion

$$
\ddot{\theta}_{i}=F_{i}=-\frac{\epsilon}{N} \sum_{j=1}^{N} \sin \left(\theta_{i}-\theta_{j}\right),
$$

where $F_{i}$ is the force on the $i$ th particle. They correspond to a system of fully coupled pendula. Let us introduce the total spin vector

$$
\mathbf{M}=\left(M_{x}, M_{y}\right)=\frac{1}{N} \sum_{j=1}^{N} \mathbf{m}_{i}
$$

and the phase of $\mathbf{M}$,

$$
\tan \phi=\frac{M_{y}}{M_{x}}
$$

with $-\pi<\phi \leq \pi$. After these definitions, Eq. (3) can be reexpressed as that of a perturbed pendulum

$$
\ddot{\theta}_{i}=-\epsilon M \sin \left(\theta_{i}-\phi\right) \text {. }
$$

Both $M$ and $\phi$ depend on time through the coordinates $\theta_{i}$. Therefore the motion of each particle is determined self-consistently by the time evolution of the spin vector $\mathbf{M}$, which itself depends on the motion of all the particles. We study two kinds of systems, according to the sign of $\epsilon$. When $\epsilon>0$ (see Sec. III), the potential is attractive and the ground state is reached when all the particles have the same position on the circle. In this case the dynamics corresponds to that of a ferromagnetic model. An example of a low energy state is shown in Fig. 1(a), where we represent the unit circle together with the coordinates $\theta_{i}$ of all the $N$ particles. When $\epsilon<0$ the potential becomes 
repulsive. Model (1) then describes the dynamics of an antiferromagnetic system (see Sec. IV). The ground state would correspond to the uniform distribution of the $N$ particles on the circle $p_{i}=0, \theta_{i}=2 \pi i / N$. This state is dynamically stable. However, as we will see, a twocluster state [see Fig. 1(b)] forms spontaneously at very small energy, or it can be excited by an initial modulation of particle density at a larger energy. In both the ferromagnetic and the antiferromagnetic cases there exists a large kinetic energy (large temperature) state in which each particle visits uniformly all the circle, making $\mathbf{M}=\mathbf{0}$. Thermodynamically, the ferromagnetic model has a second order phase transition with order parameter $\mathbf{M}$; while in the antiferromagnetic case the free energy is constant (see Sec. II).
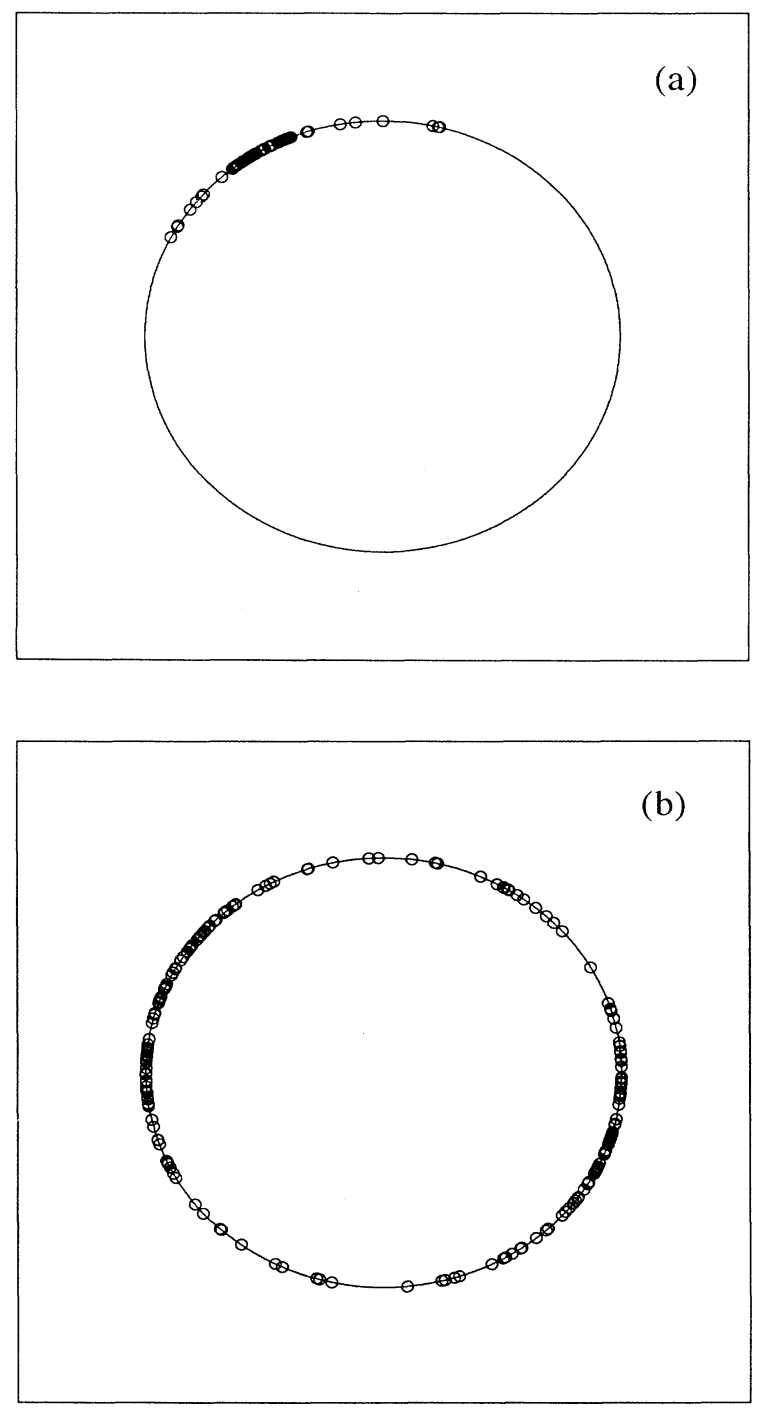

FIG. 1. The particles $\pi<\theta_{i} \leq \pi$ are all represented by small open circles on the unit circle. (a) Clustering state with $U=0.05, N=1000, \epsilon=1$. (b) Two-cluster state with $U=-0.499, N=1000, \epsilon=-1$, very close to the ground state.
Hamiltonian (1) may also be related to other types of models with long-range interactions. Indeed, the potential energy in (1) is the first term in a Fourier expansion of the potential energy of a system composed of $N$ identical parallel sheets with an infinite extent in the $(y, z)$ plane, moving along the $x$ axis with periodic boundary conditions ( $L$ being the length of the periodic line) and with a constant acceleration between two crossings. The equations of motion of this system are

$$
\ddot{x}_{i}=F_{i}=-\alpha \sum_{j=1}^{N} \sum_{n=0}^{+\infty} \frac{1}{k_{n}} \sin \left(k_{n}\left(x_{i}-x_{j}\right)\right)
$$

where $x_{i}$ is the position of the $i$ th sheet along the $x$ axis and $k_{n}=2 \pi(2 n+1) / L$. In the model of charged sheets (CSM) $[3,4]$

$$
\alpha=-4 \sigma^{2} /\left(\mu L \epsilon_{0}\right)
$$

$\sigma$ being the surface charge density, $\mu$ the mass density, and $\epsilon_{0}$ the permittivity of the vacuum. In the model of gravitational sheets (GSM) [3,5]

$$
\alpha=16 \pi G \mu / L
$$

where $G$ is the Newton constant. The analogy of Eq. (7) with that for the HMF, Eq. (3), is evident. The dynamics is modified by the presence of higher Fourier components in the force, but we argue that several large scale collective properties are not strongly affected by adding interactions on smaller spatial scales, although relaxation properties are modified. We show in Fig. 2 the force function for the GSM, together with the one mode HMF force and the one corresponding to adding one further mode; a particle will feel strong differences among these force functions only when it is visiting the regions at the border of the plateau. However, the relation among the dynamics of the CSM, GSM, and of the $\mathrm{HMF}$ is subtle; similar phenomena are observed by looking at $\mathbf{M}=\left(\sum_{i}^{N} \cos \left(k_{0} x_{i}\right), \sum_{i}^{N} \sin \left(k_{0} x_{i}\right)\right) / N$ (which corresponds to the first mode of the electrostatic field), but differences in the low energy spatial distribution of particles are present. Numerical simulations show the presence of a "clustering state" for the GSM [3] at small energy as in the ferromagnetic HMF. A "declustering" transition is present as energy is increased. On the contrary, for the CSM the averaged $\mathbf{M}$ vanishes as in the antiferromagnetic HMF. However, one obtains long-time persistent oscillations of $\mathbf{M}$ if one initially excites the system with a nonuniform particle density distribution [6].

An analogy of ferromagnetic Hamiltonian (1) with models of self-gravitating systems has been proposed in Ref. [7]. A Vlasov-Poisson equation has been introduced, which reveals an instability at a critical temperature; below this temperature a "clustering" state is stable. A discrete time version of model (1) has been studied in Ref [8]. It reduces to a system of globally coupled symplectic maps, and shows a "clustering" phase in the small momentum range for the ferromagnetic case.

Model (1) may be considered as a microcanonical simulation of the mean-field $X-Y$ model. Analogously to 


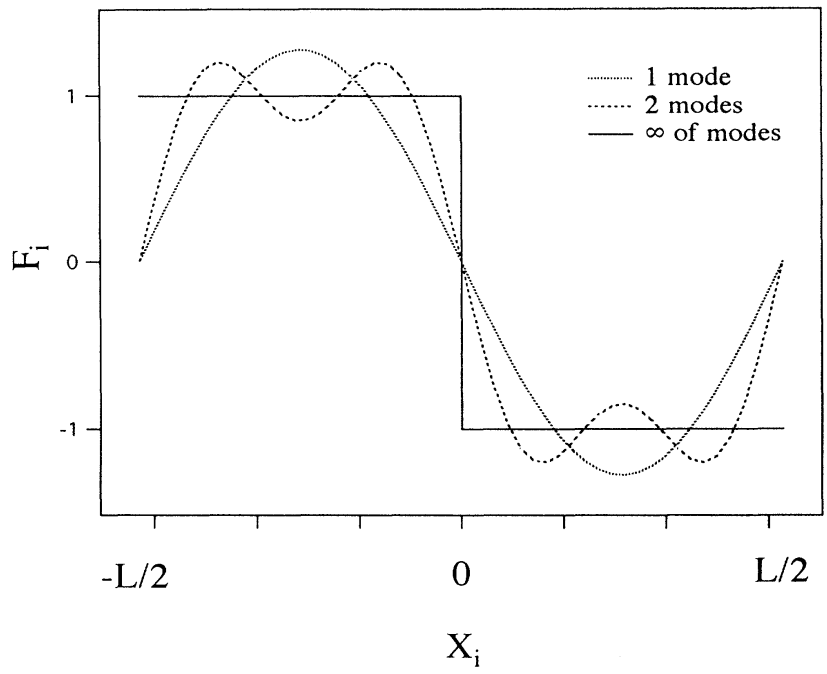

FIG. 2. Behavior of the force $F_{i}$ in formula (7) with one, two, and an infinity of Fourier components, generated by a particle at position $x_{i}=0$.

what is done in Ref. [9] for the Ising model, we add a kinetic energy term through the conjugate variable of the angle $\theta_{i}$. As for the Ising microcanonical dynamics we have strong violations of ergodicity, but still the dynamics reproduces some features of the equilibrium solution, as we will see in the following.

There are also physical justifications for adding a kinetic energy term. One is the presence of single ion anisotropy terms of the kind $S_{z}^{2}$ in magnetic systems [10], which in the classical limit play the role of a fictitious kinetic energy for the dynamics of the $X-Y$ components of the spin vector. Another is the more simple fact that the dynamics of the $X-Y$ model can be rewritten in the semiclassical approximation, valid for large value of the spin, exactly in the form (1), introducing appropriate conjugate variables.

The equations of motion of our model (6) have also a close form to those of Compton free electron laser (FEL) amplifiers [11]. Also the phenomenology is quite close; at some critical value of the average kinetic energy the electrons begin to transfer their energy coherently to the radiation field. The field amplitude plays the role of our order parameter (4). The main difference from our model is that in the FEL case the field is not generated selfconsistently by the motion of the electrons, but imposed externally, with its own equation of motion.

\section{EQUILIBRIUM STATISTICAL MECHANICS}

We present in this section the calculation of the free energy and of some relevant observables (e.g., M) of model (1) in the canonical ensemble. The energy vs temperature relation is also derived, to establish the connection with the microcanonical ensemble in which the numerical simulations are performed. The exact equilibrium solution of model (1) results from a straightforward application of the Hubbard-Stratonovich trick. The partition function of model (1) is

$$
Z=\int \prod_{l=1}^{N} d p_{l} d \theta_{l} \exp (-\beta H)
$$

where the integration is extended over all the phase space and $\beta$ is the inverse temperature, $\beta=1 /\left(k_{B} T\right)$. $Z$ factorizes into a kinetic contribution

$$
Z_{K}=\left(\frac{2 \pi}{\beta}\right)^{\frac{N}{2}}
$$

and a potential one

$$
Z_{V}=\exp \left(-\frac{\beta \epsilon N}{2}\right) J
$$

with

$$
J=\int_{-\pi}^{\pi} \prod_{l=1}^{N} d \theta_{l} \exp \left(\frac{\beta \epsilon}{2 N} \sum_{i, j=1}^{N} \cos \left(\theta_{i}-\theta_{j}\right)\right) .
$$

$J$ can be reexpressed as

$$
J=\int_{-\pi}^{\pi} \prod_{l=1}^{N} d \theta_{l} \exp \left[\frac{\beta \epsilon}{2 N}\left(\sum_{i=1}^{N} \mathbf{m}_{i}\right)^{2}\right]
$$

with $\mathbf{m}_{i}$ defined in (2). We treat only the case $\epsilon>0$ in full detail. The negative $\epsilon$ case is straightforward after some simple analytic extensions. The HubbardStratonovich transformation in the $\mu>0$ case reads

$$
\exp \left(\frac{\mu}{2} \mathbf{x}^{2}\right)=\frac{1}{\pi} \int_{-\infty}^{+\infty} \int_{-\infty}^{+\infty} d \mathbf{y} \exp \left(-\mathbf{y}^{2}+\sqrt{2 \mu} \mathbf{x} \cdot \mathbf{y}\right)
$$

where $\mathbf{y} \in \mathbf{R}^{2}$. Then Eq. (13) yields

$$
\begin{aligned}
J= & \frac{1}{\pi} \int_{-\pi}^{\pi} \prod_{l=1}^{N} d \theta_{l} \int_{-\infty}^{+\infty} \int_{-\infty}^{+\infty} d \mathbf{y} \\
& \times \exp \left(-\mathbf{y}^{2}+\sqrt{2 \mu} \sum_{l=1}^{N} \mathbf{m}_{l} \cdot \mathbf{y}\right)
\end{aligned}
$$

with $\mu=\beta \epsilon / N$. Exchanging the two integrals, one can factorize the integration over the coordinates of the $N$ particles. Then, performing this integration and rescaling $\mathbf{y} \rightarrow \mathbf{y} \sqrt{N / 2 \beta \epsilon}$, one gets

$$
\begin{aligned}
J=\frac{1}{\pi} & \frac{N}{2 \beta \epsilon} \int_{-\infty}^{+\infty} \int_{-\infty}^{+\infty} d y \\
& \times \exp \left(-N\left[\frac{y^{2}}{2 \beta \epsilon}-\ln \left(2 \pi I_{0}(y)\right)\right]\right),
\end{aligned}
$$

where $y$ is the modulus of $\mathbf{y}$ and $I_{0}$ is the zero order modified Bessel function. The free energy is given by

$$
F=-\lim _{N \rightarrow \infty}\left(\frac{1}{\beta N} \ln Z\right)
$$


with $Z$ the partition function given in (10). Then, using the saddle point method,

$$
\begin{aligned}
-\beta F= & \frac{1}{2} \ln \left(\frac{2 \pi}{\beta}\right)-\frac{\epsilon \beta}{2} \\
& +\max _{y}\left(-\frac{y^{2}}{2 \beta \epsilon}+\ln \left(2 \pi I_{0}(y)\right)\right) .
\end{aligned}
$$

Since $F$ depends only on the modulus $y$, there is an infinity of minima reached when

$$
\frac{y}{\beta \epsilon}-\frac{I_{1}}{I_{0}}(y)=0 \text {. }
$$

This is a consistency equation whose minimal free energy solution is $y=0$, corresponding to a vanishing magnetization, for $\beta \epsilon<2$. For $\beta \epsilon>2$ the solution is a nonvanishing $\beta$-dependent value of $y$, which can be determined numerically. The magnetization is given by the ratio of Bessel functions $I_{1} / I_{0}$ in Eq. (16) evaluated at the solution of the same consistency equation, as it can be proven by solving the external field dependent free energy and computing its derivative for vanishing field (a calculation that we omit here). However, this result can be easily guessed considering that this ratio is bounded by +1 and -1 , reaching these values for $y=+\infty$ and $y=-\infty$, respectively. The phase transition is second order and the critical exponent is the classical one $(1 / 2)$. This can be seen by Taylor expanding (16) and solving for $y$. In Fig. 3 the theoretical curve representing the magnetization vs energy $U=H / N$ is the dashed line.

The internal energy (or energy density) $U$ is given by

$$
U=\frac{\partial(\beta F)}{\partial \beta}
$$

which yields

$$
U=\frac{1}{2 \beta}+\frac{\epsilon}{2}\left(1-M^{2}\right)
$$

and is represented by the dashed line in Fig. 4. When

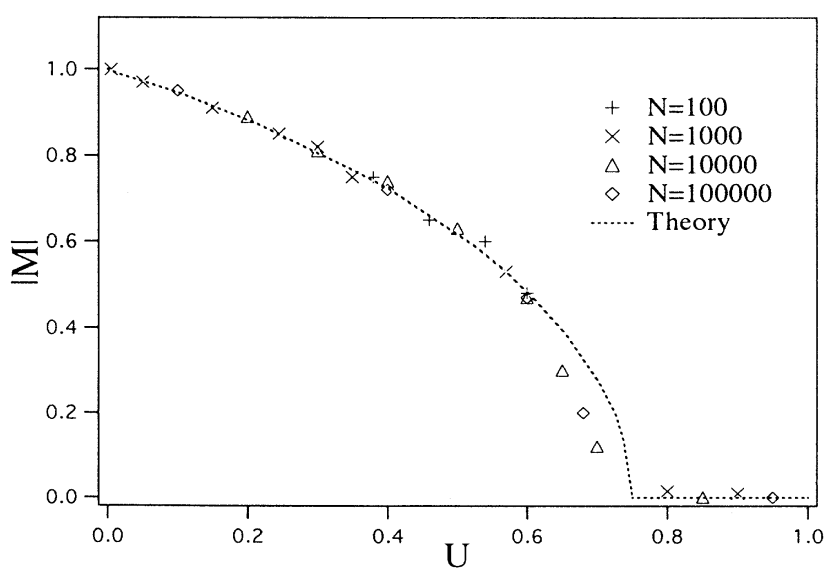

FIG. 3. Modulus of the magnetization in formula (4) as a function of the energy $U=H / N$. The dashed line is the theoretical equilibrium result, the points are results of numerical experiments performed in the microcanonical ensemble.

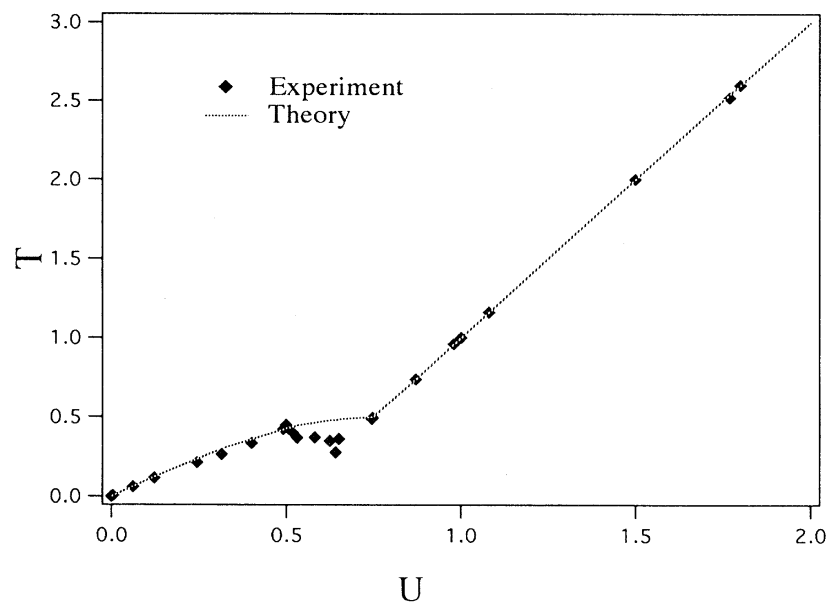

FIG. 4. Temperature (twice the averaged kinetic energy) as a function of the energy $U=H / N$. The dotted line is the theoretical equilibrium result, the points are results of numerical experiments in the microcanonical ensemble.

$\epsilon<0$ the procedure applied for the $\epsilon>0$ case can be easily repeated, but the unique solution of the consistency equation is $\mathbf{y}=\mathbf{0}$ for any $\boldsymbol{\beta}$. Therefore, the antiferromagnetic $\mathrm{HMF}$ has no phase transition, the magnetization remains zero. The free energy and the internal energy are those in formulas (15) and (17) with $M=0(y=0)$.

\section{NONEQUILIBRIUM DYNAMICS OF THE FERROMAGNETIC HMF}

To integrate numerically model (1) we use a second order symplectic scheme (leap-frog), choosing a time step of the order of $1 \%$ of the characteristic linear pulsation $\sqrt{\epsilon}$, which gives energy fluctuations $\mathcal{O}\left(10^{-4}\right)$. Since the interactions among all particles in HMF are the same, the size of the algorithm is $\mathcal{O}(N)$ instead of $\mathcal{O}\left(N^{2}\right)$, provided the total spin vector $\mathbf{M}$ is evaluated before computing the force. We study the time evolution of large $N$ systems (typically $N \approx 10000$ ). To be initially in nonequilibrium, the runs all begin with a water-bag $[4,12]$ velocity distribution, corresponding to a constant distribution on the momentum support $[-\alpha, \alpha]$, either localized or uniform over the coordinates.

\section{A. Identification of the dynamical regimes}

As described in Sec. II for equilibrium states, subcritical and supercritical situations occur depending on the value of the internal energy, with a critical energy $U_{c}=(3 / 4) \epsilon$ (in all the numerical experiments described in the following $\epsilon=1$ ). We present in Fig. 4 the numerical measurements of the temperature $T=2\langle K(t)\rangle / N$, where $K$ is the kinetic energy defined in Eq. (1) and \langle\rangle denotes time averaging, as a function of $U$. Both Fig. 3 and Fig. 4 clearly confirm the presence of a critical energy $U_{c}$ where magnetization $M$ vanishes and the $T / U$ 
relation becomes linear. To get more information on the dynamical properties of the subcritical regime, we introduce a distinction between two classes of particles: high energy (HEP) and low energy (LEP) particles.

The separatrix width of the perturbed pendulum motion of Eq. (6) is $2 \sqrt{\epsilon M}$ and its energy $\epsilon(1+M)$. Particles with an energy $e_{i}>\epsilon(1+M)$,

$$
e_{i}=\frac{p_{i}^{2}}{2}+\frac{\epsilon}{N} \sum_{j=1}^{N}\left(1-\cos \left(\theta_{i}-\theta_{j}\right)\right)
$$

which we call high energy particles (HEP), will visit all the circle while those having an energy $e_{i}<\epsilon(1+M)$, the low energy particles (LEP), will not. These latter are trapped in the resonance bounded by the separatrix. We use the notion of separatrix and the identification of the two populations of particles, HEP and LEP, to define the trapping probability $p(U)$, as the fraction $N_{\text {LEP }} / N$, where $N_{\text {LEP }}$ is the number of LEP, after the system has reached its equilibrium state. This quantity is the analog of the "activity" parameter introduced in Ref. [13]. We represent in Fig. 5 the energy dependence of $p(U) . p(U)$ remains close to unity up to $U=U_{b}$, with $U_{b} \approx 0.3$, as shown by the intersection of the two straight segments in Fig. 5. As soon as $U>U_{b}, p(U)$ decreases, reaching zero at $U=U_{c}$. We call the regime $U \in\left[0, U_{b}\right]$ subcritical bounded regime, because a cluster of particles with no center of mass kinetic energy forms spontaneously from the initial condition, as we will comment in the following; this also corresponds to trapping of the majority of the particles in one resonance. For $U \in\left[U_{b}, U_{c}\right]$ the center of mass of the cluster drifts. We call this regime the subcritical translational regime. In the supercritical regime, the separatrix width shrinks to zero, giving $p(U)=0$. The kinetic energy is large enough for most of the particles to visit the circle.

In the subcritical bounded regime $T / U \sim 1$ and nu-

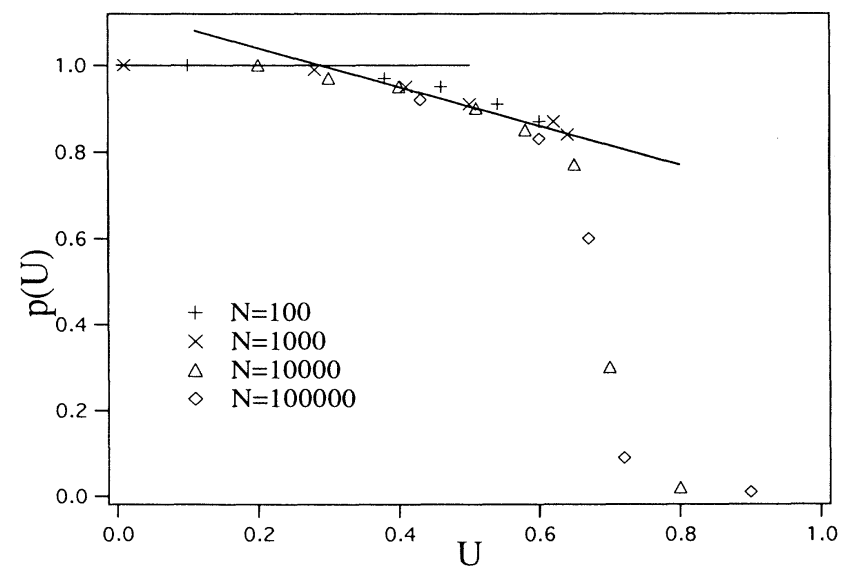

FIG. 5. Trapping probability $p(U)$ as a function of the energy $U$. The horizontal straight segment is drawn to show that in the bounded subcritical regime $p(U) \approx 1$. The other straight segment guides the eyes through decreasing values of $p(U)$ in the translational subcritical regime. The intersection between the two segments determines $U_{b} \approx 0.3$. merical results are in good agreement with the theoretical equilibrium calculation of Sec. II. The dynamics is dominated by potential energy; all the particles linearly oscillate in the neighborhood of a constant global phase $\phi$ with pulsation $\sqrt{\epsilon M}$ [see Eqs. (6)]; no drift in $\phi$ is observed and the system forms a single still cluster (see Fig. 1). The phase $\phi$ shows time spectral properties similar to those of a single particle. In the subcritical translational regime the averaged kinetic energy shows a decrease with energy, indicating a negative specific heat. This result might be a nonequilibrium effect, as we will argue. However, one should also observe that our model does not verify the subadditivity condition [2], which in turn implies a violation of convexity; thus, this result might also be relevant as far as equilibrium properties are concerned. In the subcritical translational regime the phase $\phi$ in Eq. (6) drifts; this corresponds to a motion of the cluster on the circle (we will comment on this in the following). In the supercritical regime $\left(U>U_{c}\right)$, the agreement with the statistical predictions is again good.

\section{B. Phase dynamics in the translational regime}

For energies $U_{b} \leq U \leq U_{c}$ the system has energy enough to generate a sizeable fraction of HEP. As an example, we represent in Fig. 6 the time evolution of the phase $\phi(t)$ together with the number of HEP, when this latter is zero or one. The phase has a drift only when at least one HEP is present. When no HEP is present, on the other hand, $\phi$ fluctuates around a constant mean value.

Let us consider the one-particle distribution function $f(\theta, p, t)$, defined as usual through the probability $d P=$ $f d \theta d p$ of finding a particle with a coordinate in the interval $[\theta, \theta+d \theta]$ and momentum in $[p, p+d p]$ at time $t[14]$ in the continuum $N \rightarrow \infty$ limit. We have numerically computed the reduced distribution $n(\theta, t)=\int f(\theta, p, t) d p$ for $N=10000$. Figure 7 clearly shows the presence

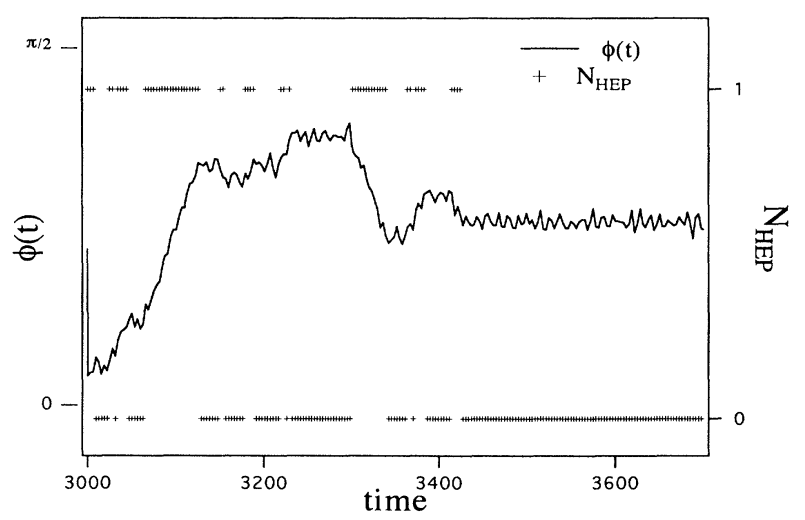

FIG. 6. We plot the phase $\phi$ vs time with a full line. The crosses are set to 1 if there is one untrapped particle, and to zero if all particles are trapped. This shows that phase drift is present only when at least one particle is untrapped. The scale on the left axis is for the phase. $N=100, U=0.3$. 


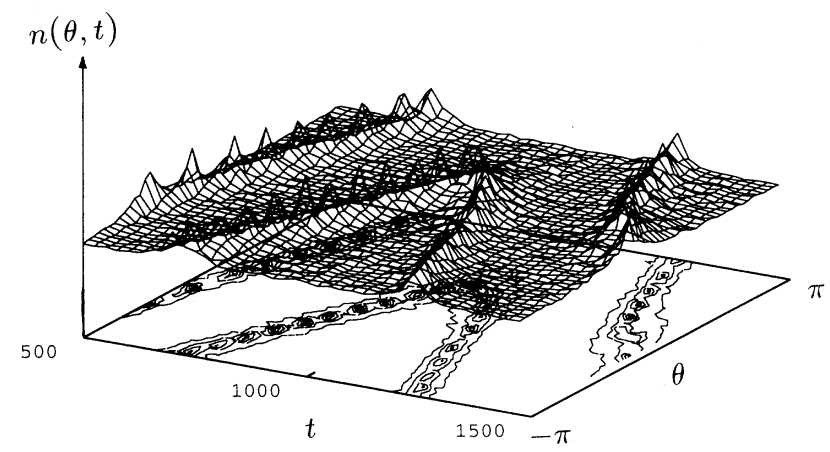

FIG. 7. Plot of the distribution function $n(\theta, t) . U=0.4$, $N=10000$. The contour plot is also drawn in the $(t, \theta)$ plane.

of a single drifting cluster of particles. The drift signals a collective motion of all the particles and yields in $\left(M_{x}, M_{y}\right)$ plane a circular shape with radius $M$ given in formula (16) whose thickness scales as $1 / \sqrt{N}$ (see Fig. 8).

The drift of the phase is a consequence of the conservation of total momentum and of the existence of a separatrix. Let us explain this simple fact. Let $\zeta(t)$ be the window averaged phase (the size of the window is large with respect to $1 / \sqrt{\epsilon M}$ ), and define the coordinates in the moving frame with origin in $\zeta$,

$$
\begin{aligned}
\theta_{i}(t) & =\zeta(t)+\eta_{i}(t) \\
\phi(t) & =\zeta(t)+\psi(t)
\end{aligned}
$$

where

$$
\tan \psi(t)=\frac{\sum_{i} \sin \left(\eta_{i}\right)}{\sum_{i} \cos \left(\eta_{i}\right)} .
$$

As suggested by numerical simulations, the average phase

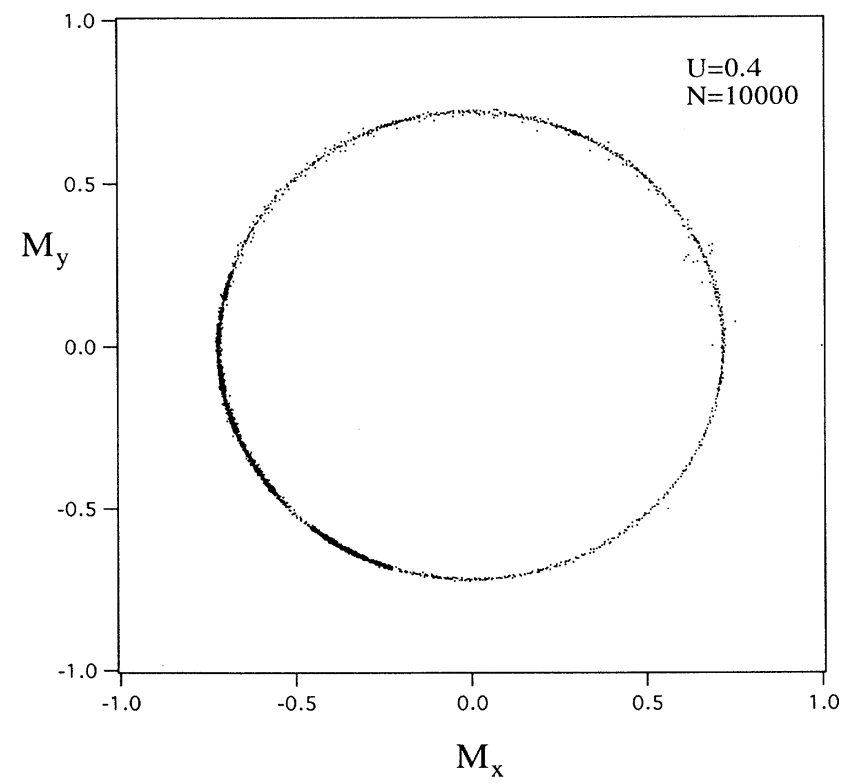

FIG. 8. $M_{y}$ vs $M_{x}$ for $U=0.4$ and $N=10000$ after a transient. The experiment is the same as in Fig. 7. $\zeta(t)$ slowly varies on a time scale $\mu \gg 1 / \sqrt{\epsilon M}$. Conservation of total momentum implies

$$
\langle\dot{\zeta}\rangle=-\frac{1}{N} \sum_{i=1}^{N}\left\langle\dot{\eta}_{i}\right\rangle
$$

if we restrict, without loss of generality, to an initially vanishing total momentum. Here, \langle\rangle represents an average over the time interval $\mu$. If a separatrix exists, then only the HEP have a nonzero slowly varying average momentum $p_{i}-\dot{\zeta}$, while the average contribution of LEP to the total momentum is zero; thus we can restrict the sum on the right-hand side of Eq. (21) to HEP. Since also $\dot{\zeta}$ is slowly varying over $\mu$,

$$
\dot{\zeta}=-\frac{N_{\mathrm{HEP}}}{N_{\mathrm{LEP}}}\langle p\rangle_{\mathrm{HEP}}
$$

where $\langle p\rangle_{\text {HEP }}$ is the time averaged momentum of HEP.

In Fig. 9 we show a numerical check of Eq. (22). In the low energy subcritical bounded regime, Eq. (22) is trivially the equality between two vanishing quantities, while in the supercritical regime the hypothesis that $\zeta$ slowly varies is no longer valid (instead the time average of $\dot{\zeta}$ is zero in this regime).

A consequence of this slow drift of the phase is the appearance of two resonances centered on $(\zeta, \pm \dot{\zeta})$ in the $(\theta, p)$ plane of width $2 \sqrt{\epsilon M}$, which strongly overlap since $\dot{\zeta} \sim 1 / \sqrt{N}$, as it has been observed numerically in the time intervals where $\dot{\zeta}$ is constant in time (see Fig. 10); the two resonances are not present at the same time (an example is given in Fig. 11). The rescaled average drift $N^{1 / 2} \dot{\zeta}$ grows with $U$ approaching the critical energy (see again Fig. 10). A chaotic sea in the narrow domain $\mathcal{O}(1 / \sqrt{N})$ swept by the motion of the separatrix is present. Moreover, on much longer time scales weak chaos reaches also the center of the resonances, due to the effective superposition of the two resonances [15]; it is on these time scales that we expect agreement between equilibrium averages and time averages.

Both in the subcritical bounded and in the translational regimes, $n(\theta, t)$ is nonuniform at each time (corresponding to the presence of a localized pattern, the cluster of particles). In the subcritical translational regime, because of the collective motion of the cluster the time averaged distribution $\langle n\rangle$ becomes uniform. When the initial condition of the system is very far from equilibrium, as it is the case in Fig. 11, where initially momenta are distributed in a water bag and coordinates are lumped at a given value, the dynamics develops initially a spiral structure in the Boltzmann $\mu$ space [a snapshop of the coordinates and momenta $\left\{\theta_{i}, p_{i}\right\}$ of all the particles into the $(\theta, p)$ plane] [14]. The spiral structure was already known to exist in the dynamics of GSM [16]. This structure is characterized by a differential rotation and by a global motion of its center with a speed given by Eq. (22). 


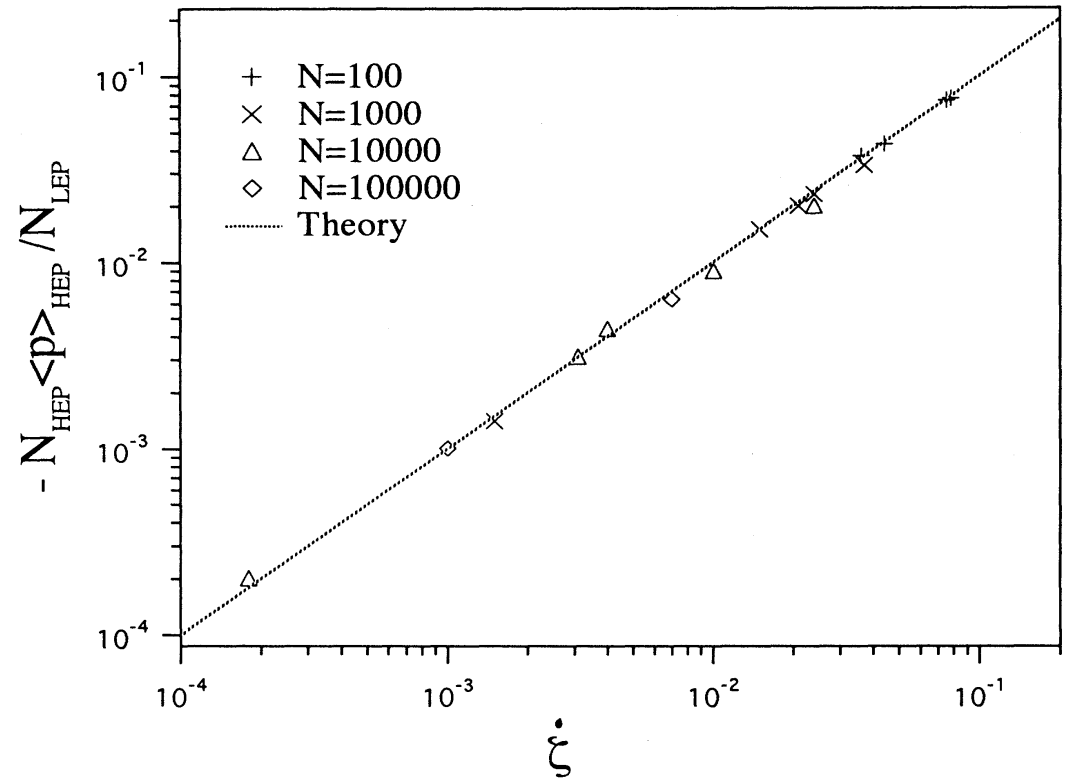

FIG. 9. Relation between the averaged momentum of the high energy particles and the phase drift velocity (22). The dotted line is the theoretical prediction and the points are time averages obtained in numerical experiments. Due to time reversal invariance the same relation holds for negative momentum. The energies vary in the range $U_{b}<U<U_{c}$.

\section{Relaxation time in the supercritical regime}

In the supercritical regime, the particles move almost freely. The clusters involve a negligible fraction of the particles and have a short lifetime, and the phase $\phi$ has zero average. The spatial distribution of the particles is almost uniform on the circle and the functions $M_{x}(t)$ and $M_{y}(t)$ take most of their values in the fluctuation range $\mathcal{O}(1 / \sqrt{N})$. In order to estimate numerically the typical relaxation time $\tau_{f}$ of the initial water-bag velocity distribution to the Maxwellian one, we identify in the system two families of particles at time 0: SPO (slow particles) and FPO (fast particles). SPO groups the $N / 2$ particles having initially a momentum $\left|p_{i}\right|<\alpha / 2$ and FPO those having initially a momentum $\left|p_{i}\right|>\alpha / 2$, where $\alpha$ is the width of the initial water-bag momentum distribu-

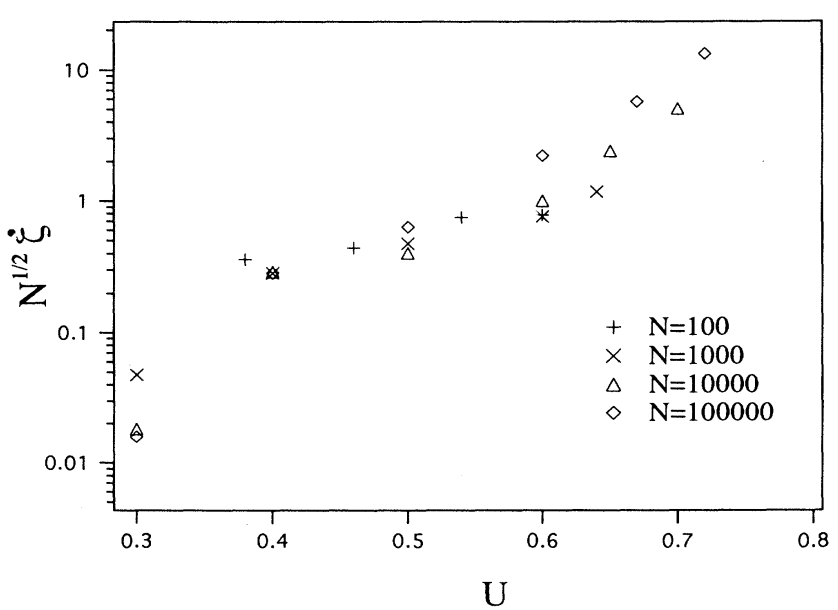

FIG. 10. $N^{1 / 2} \dot{\zeta}$ as a function of $U$. The data collapse prove the scaling of $\dot{\zeta}$ as $N^{-1 / 2}$ for various values of $U$. tion. One must remark that this partition has a different nature than the one introduced previously between HEP and LEP; in the supercritical regime the separatrix shrinks $(\mathbf{M}=\mathbf{0})$, making all particles HEP. Therefore the partition into SPO and FPO is inside the HEP group.

The relaxation time is determined through the time evolution of

$$
\begin{aligned}
& K_{\mathrm{SPO}}(t)=\frac{1}{2 K} \sum_{i \in \mathrm{SPO}} p_{i}^{2} \\
& K_{\mathrm{FPO}}(t)=\frac{1}{2 K} \sum_{i \in \mathrm{FPO}} p_{i}^{2}
\end{aligned}
$$

where $K_{\mathrm{SPO}}(t)$ and $K_{\mathrm{FPO}}(t)$ correspond to the kinetic energy fraction carried by the slow and fast particles, re-

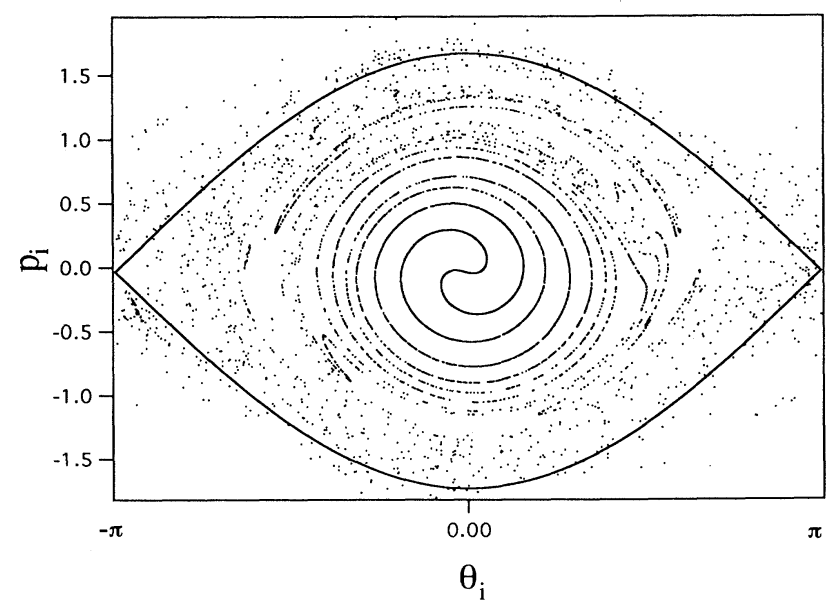

FIG. 11. Boltzmann $\mu$ space for $U=0.4$ and $N=10000$ in the transient state, together with the separatrix of the perturbed pendulum of Eq. (6) (full line). The experiment is the same as in Fig. 7 and Fig. 8. 
TABLE I. Correspondence among physical parameters and behaviors of HMF, CSM, and GSM. $R$ is the radius of the circle in the HMF ( $R=1$ in our case).

\begin{tabular}{|c|c|c|c|}
\hline & HMF & CSM & GSM \\
\hline linear pulsation & $\omega_{\mathrm{HMF}}=\sqrt{|\epsilon|}$ & $\begin{array}{c}\omega_{\mathrm{pl}}=\sqrt{\frac{4 \sigma^{2} \mathcal{N}}{\mu \epsilon_{o}}} \\
\text { Plasma pulsation }\end{array}$ & $\begin{array}{c}\omega_{J}=\sqrt{16 \pi G \mu \mathcal{N}} \\
\text { Jeans pulsation }\end{array}$ \\
\hline temperature & $T=2 R\langle K\rangle$ & $T=\left\langle\dot{x}^{2}\right\rangle=v_{t}^{2}$ & $T=\left\langle\dot{x}^{2}\right\rangle=v_{t}^{2}$ \\
\hline screening length & $\lambda_{\mathrm{HMF}}=R \sqrt{\frac{T}{|\epsilon|}}$ & $\lambda_{D}=\frac{v_{t}}{\omega_{\mathrm{p} 1}}$ & $\lambda_{J}=\frac{v_{t}}{\omega_{J}}$ \\
\hline $\begin{array}{l}\text { collective } \\
\text { behavior }\end{array}$ & $\frac{T}{|\epsilon|}<1$ & $\begin{array}{c}\lambda_{D} \ll \lambda \\
\text { Langmuir modes }\end{array}$ & $\begin{array}{l}\lambda_{J} \ll \lambda \\
\text { Clusters }\end{array}$ \\
\hline $\begin{array}{l}\text { grain } \\
\text { parameter }\end{array}$ & $g_{\mathrm{HMF}}=\frac{2 \pi}{N} \sqrt{\frac{|\epsilon|}{T}}$ & $g_{\mathrm{CSM}}=\frac{1}{\mathcal{N} \lambda_{D}}$ & $g_{\mathrm{GSM}}=\frac{1}{\mathcal{N} \lambda_{J}}$ \\
\hline
\end{tabular}

spectively. $K$ is the total kinetic energy. As the system evolves, the two populations SPO and FPO mix themselves exchanging their kinetic energies and the quantities $K_{\mathrm{SPO}}(t)$ and $K_{\mathrm{FPO}}(t)$ relax to $1 / 2$.

In the study of the GSM the relaxation of the quantities defined in Eqs. (23) and (24) is also considered [12]. A relaxation time

$$
\tau_{\mathrm{GSM}}=\frac{1}{g_{\mathrm{GSM}} \omega_{J}}
$$

is introduced, which is defined in terms of the "grain" parameter [17]

$$
g_{\mathrm{GSM}}=\frac{1}{\mathcal{N} \lambda_{J}}
$$

where $\mathcal{N}=N / L$ is the density of sheets on the periodic segment of length $L$ and $\lambda_{J}=v_{t} / \omega_{J}, v_{t}$ being the average squared speed of the particles obeying Eq. (7) and $\omega_{J}=\sqrt{16 \pi G \mu \mathcal{N}}$ the Jeans pulsation (see also Sec. V and Table I). As discussed in the Introduction, the dynamics of the ferromagnetic HMF resembles that of GSM, if only large scale behavior is studied. In Sec. V we discuss in detail how to establish a correspondence between the physical parameters introduced in the two models; this

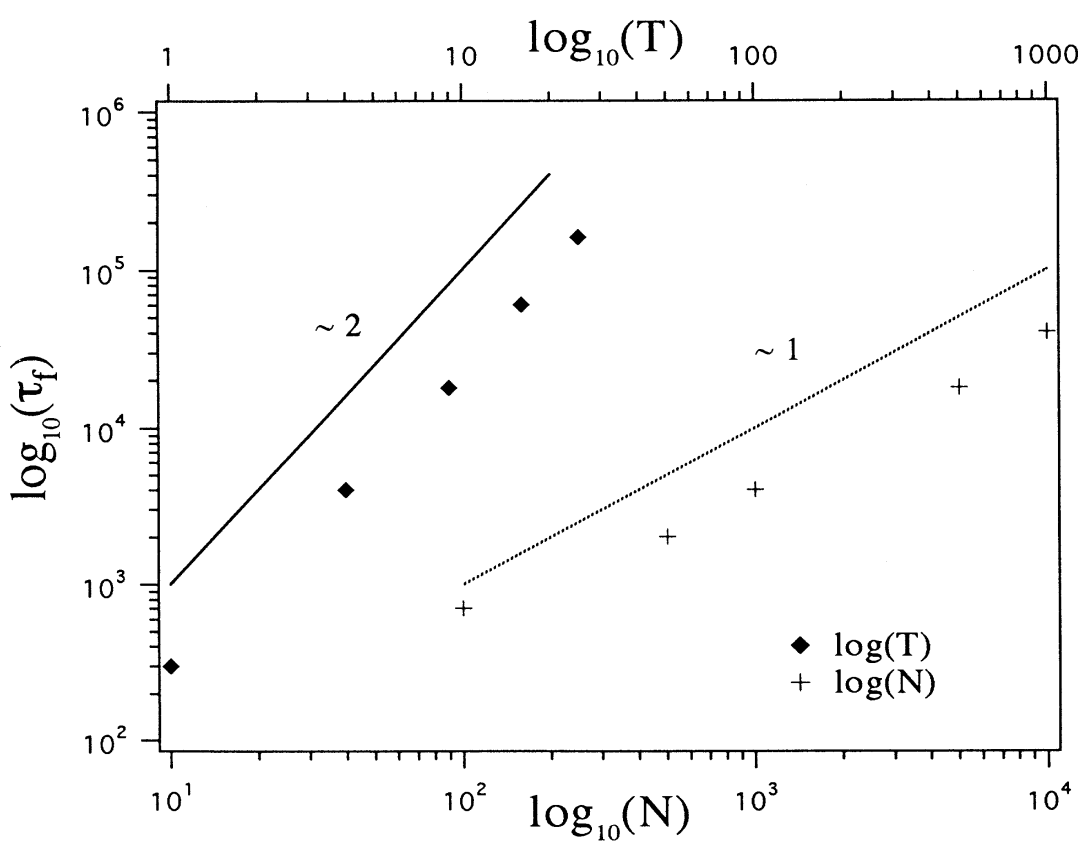

FIG. 12. Relaxation time $\tau_{f}$ as a function of $N$ with $T=1$ (crosses), and vs $T$ with $N=10000$ (full diamonds). In the figure the theoretical slope 1 , corresponding to the $N$ dependence, is also reported (dotted line), together with the slope 2 related to the $T$ dependence (full line). 


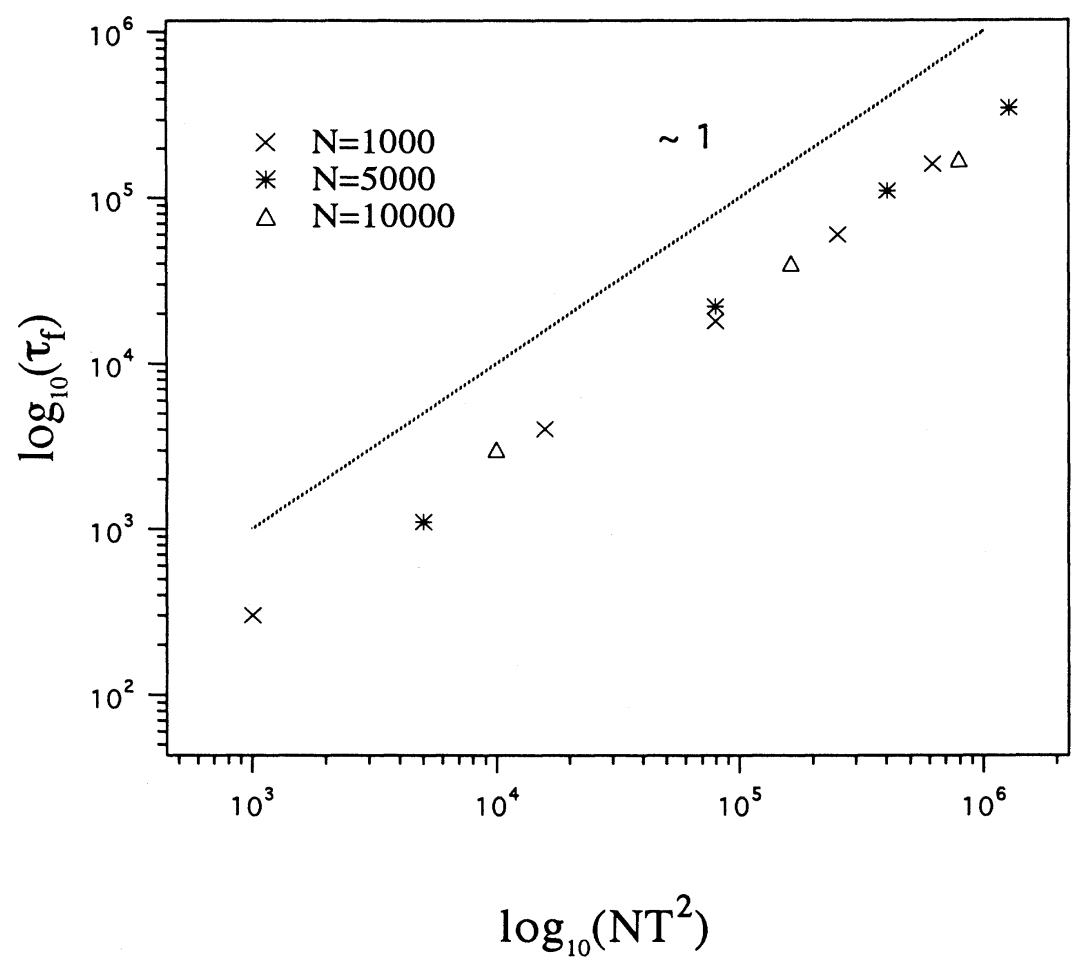

FIG. 13. Relaxation time $\tau_{f}$ as a function of $N T^{2}$. The points are well fitted by a slope 1.

correspondence is summarized in Table I. According to this table, it is easy to derive that the relaxation time scale of the HMF should have the following dependence on $N$ and $T$ :

$$
\tau_{\mathrm{HMF}} \sim N \sqrt{T}
$$

once one has introduced the analogous "grain" parameter for the HMF (see again Table I). We show in Fig. 12 the relaxation time $\tau_{f}(\sigma)$, defined as the time required for $K_{\mathrm{SPO}}$ to reach a fixed arbitrary value $\sigma(\sigma=1 / 4$ typically) as a function of $N$. The linear dependence is in agreement with the prediction of Eq. (27). However, as far as the $T$ dependence is concerned, the slope appears to be close to 2, as shown again in Fig. 12. Therefore, the relaxation to equilibrium in the HMF is much slower than the one expected from our analogy with GSM. The $N T^{2}$ dependence of $\tau_{f}$ is summarized in Fig. 13, and moreover we show in Fig. 14 the collapse of five relaxation curves of $K_{\text {SPO }}$ corresponding to different values of $N$ and $T$. After all, it is not surprising that the relaxation properties of the ferromagnetic HMF are different from those of the GSM, and it is quite remarkable that, at least as far as the $N$ dependence is concerned, we get the same scaling law.

\section{Frequency vs energy dependence}

In the subcritical bounded regime, all particles are linearly oscillating in the center of the resonance. In this linear regime one can analytically compute the spectral properties of $\mathbf{M}$. The spectrum of $M_{x}$ and $M_{y}$ has lines at frequencies $\nu_{k}=k \sqrt{\epsilon}$ with even and odd $k$, respectively.
In the subcritical translational regime the spectrum is broad due to chaos.

In the supercritical regime linear aspects again arise, since more and more particles have a ballistic motion and weakly interact among each other. Then, an initial momentum distribution changes slowly. This allows an explicit calculation of the frequency spectrum, using the Vlasov-Poisson equations [18]. The use of these equations is also justified by the fact that the interaction we consider is weak at small angle differences, implying the validity of a noncollisional treatment.

The Vlasov-Poisson equations for the HMF are

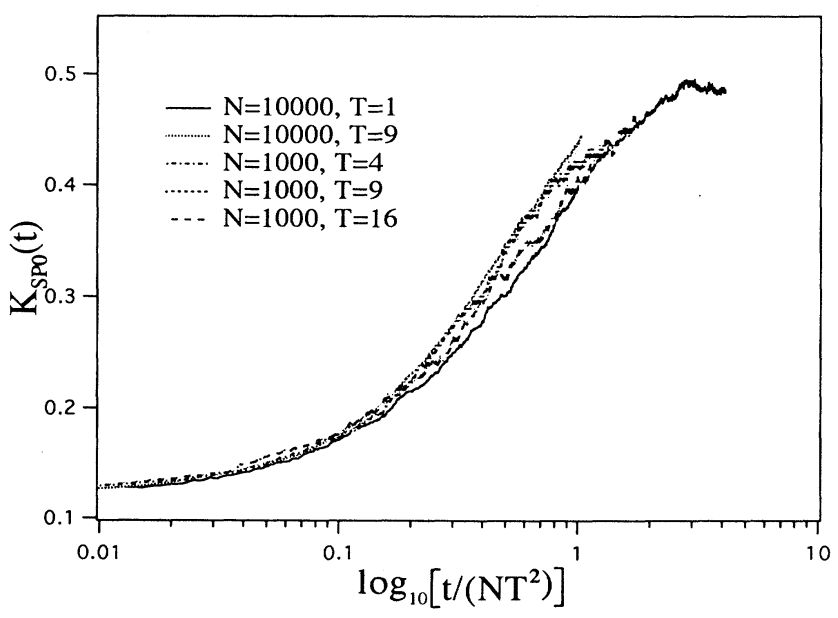

FIG. 14. Temporal evolution of the kinetic energy pertaining to the $N / 2$ low velocity particles in formula (23) with time rescaled by $\tau_{f}=N T^{2}$ with $N=1000$ and 10000 and several values of $T: T=1, T=4, T=9, T=16$. 


$$
\begin{gathered}
\frac{\partial f}{\partial t}+p \frac{\partial f}{\partial \theta}-\frac{\partial V}{\partial \theta} \frac{\partial f}{\partial p}=0 \\
\frac{\partial^{2} V}{\partial \theta^{2}}=\epsilon \int \cos \left(\theta-\theta^{\prime}\right) f\left(\theta^{\prime}, p^{\prime}, t\right) d \theta^{\prime} d p^{\prime}
\end{gathered}
$$

where $f(\theta, p, t)$ is the time dependent distribution function, and $V$ its self-consistent potential.

When the system is close to a stationary uniform state,

$$
f(\theta, p, t)=f^{(0)}(p)+f^{(1)}(\theta, p, t)
$$

where $f^{(0)}(p)$ is the stationary, uniform in $\theta$, solution of Eqs. (28) and (29) (any function of $p$ is a solution), and $f^{(1)}(\theta, p, t)$ is a small perturbation. Correspondingly, we develop the potential

$$
V(\theta, t)=V^{(0)}+V^{(1)}(\theta, t),
$$

where $V^{(0)}$ is constant because it is computed on the uniform distribution and $V^{(1)}$ is a small perturbation. Considering only the first terms in a perturbative expansion one obtains

$$
\begin{gathered}
\frac{\partial f^{(1)}}{\partial t}+p \frac{\partial f^{(1)}}{\partial \theta}-\frac{\partial V^{(1)}}{\partial \theta} \frac{\partial f^{(0)}}{\partial p}=0 \\
\frac{\partial^{2} V^{(1)}}{\partial^{2} \theta}=\epsilon \int \cos \left(\theta-\theta^{\prime}\right) f^{(1)}\left(\theta^{\prime}, p^{\prime}, t\right) d \theta^{\prime} d p^{\prime}
\end{gathered}
$$

Since $f^{(1)}$ and $V^{(1)}$ are periodic in $\theta$ we can Fourier expand them in plane waves

$$
\begin{aligned}
f^{(1)}(\theta, p, t) & =\sum_{k} \int d \omega \exp i(k \theta-\omega t) f_{k}^{(1)}(\omega, p), \\
V^{(1)}(\theta, t) & =\sum_{k} \int d \omega \exp i(k \theta-\omega t) V_{k}^{(1)}(\omega) .
\end{aligned}
$$

Inserting these expressions in Eq. (28) and solving for $f_{k}^{(1)}(\omega, p)$ one gets

$$
f_{k}^{(1)}(\omega, p)=\frac{k V_{k}^{(1)}(\omega)}{p k-\omega} \frac{\partial f^{(0)}}{\partial p}
$$

Substituting this expression of $f_{k}^{(1)}(\omega, p)$ in the Fourier expanded Eq. (33) yields for a given $\omega$ component

$$
\begin{aligned}
\sum_{k} k V_{k}^{(1)}(\omega)\left[k \exp (i k \theta)+\frac{\epsilon}{2}\left(\int \frac{d p^{\prime}}{p^{\prime} k-\omega} \frac{\partial f^{(0)}}{\partial p^{\prime}}\right)\right. \\
\left.\times\left[\exp (i \theta) \delta_{k, 1}+\exp (-i \theta) \delta_{k,-1}\right]\right]=0
\end{aligned}
$$

where $\delta_{i, j}$ is the Kronecker symbol. The only nonvanishing coefficients $V_{k}^{(1)}$ of the Fourier expansion in Eq. (37) are those corresponding to $k= \pm 1$ if the following relation is satisfied:

$$
1+\frac{\epsilon}{2} \int \frac{f^{(0)} d p}{(p \pm \omega)^{2}}=0
$$

which has been obtained after an integration by parts, supposing that $f^{(0)}(p)$ decreases sufficiently fast at large $|p|$. It is simple to prove that the values of $\omega_{ \pm 1}$ that solve Eq. (38) satisfy the relation $\omega_{1}=-\omega_{-1}$. Moreover, defining

$$
\tilde{V}_{k}^{(1)}(t)=\int d \omega \exp (-i \omega t) V_{k}^{(1)}(\omega)
$$

one can prove that

$$
\begin{aligned}
& \tilde{V}_{1}^{(1)}(t)=M_{x}-i M_{y}=\exp \left(-i \omega_{1} t\right) \bar{V}_{1}^{(1)} \\
& \tilde{V}_{-1}^{(1)}(t)=M_{x}+i M_{y}=\exp \left(-i \omega_{-1} t\right) \bar{V}_{-1}^{(1)}
\end{aligned}
$$

where $\bar{V}_{ \pm 1}^{(1)}$ are constants and $\mathbf{M}$ can be expressed in terms of the perturbed distribution function

$$
\begin{aligned}
M_{x} & =\int d \theta d p \cos (\theta) f^{(1)}(\theta, p, t), \\
M_{y} & =\int d \theta d p \sin (\theta) f^{(1)}(\theta, p, t) .
\end{aligned}
$$

We have thus formally computed the frequency spectrum of the mean field $\mathbf{M}$, although the solution of Eq. (38) is nontrivial due to the presence of Landau poles [18,19], which oblige to add a small imaginary part to the frequency. Here, we restrict ourselves to consider a normalized water-bag initial velocity distribution: $f^{(0)}(p)=$ $1 / 2 \alpha$ on the support $[-\alpha,+\alpha]$. Neglecting damping and instabilities related to the presence of Landau poles we get the frequency

$$
\omega_{ \pm 1}^{2}=3 T-\frac{\epsilon}{2}
$$

where $T=\alpha^{2} / 3$ is the temperature. Using the result of

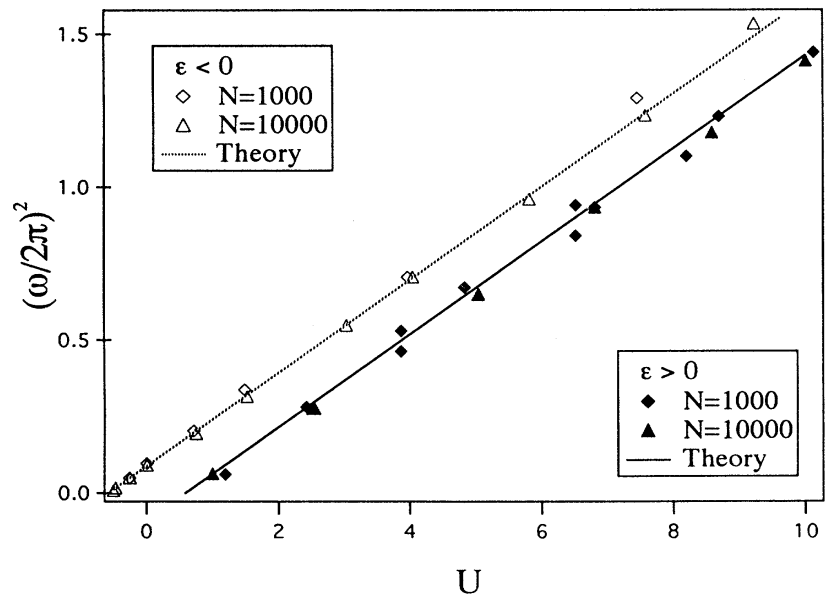

FIG. 15. Dependence of the frequency $(\omega / 2 \pi)^{2}$ given in Eq. (45) on the energy $U$. The dotted (full) line is the theoretical prediction for $\epsilon=-1(\epsilon=+1)$ and the points are the values obtained in numerical experiments $(\epsilon=-1$, open symbols; $\epsilon=+1$, full symbols). Some of the points correspond to the same value of $U$ and are due to the presence of multiple close frequencies in the spectrum, caused by the relaxation of the initial water-bag distribution. 
Eq. (17) finally yields

$$
\omega_{ \pm 1}^{2}=6 U-\frac{7}{2} \epsilon
$$

Equations (40) and (41) show that at large temperatures, if the system is prepared initially with a water-bag momentum distribution, $\mathbf{M}$ oscillates with a frequency given by Eq. (45). However, one should observe that the values taken by $\mathbf{M}$ are in the fluctuation range at high temperature [1]. Figure 15 shows the main component of the power spectrum of $\mathbf{M}$ as a function of the energy in a numerical experiment on model (1). The theoretical prediction in Eq. (45) is also reported; we observe a good agreement in the considered energy range. $\mathrm{A} \sqrt{T}$ dependence of the characteristic frequency in the supercritical regime was also derived in Ref. [1] studying the properties of a function which characterizes recurrences.

\section{ANTIFERROMAGNETIC HMF}

The statistical approach predicts the absence of phase transitions when $\epsilon<0$ (the free energy is constant). $M_{x}(t)$ and $M_{y}(t)$ take most of their values in a small $\mathcal{O}(1 / \sqrt{N})$ thermal fluctuation range band, corresponding to density fluctuations of the particles on the circle. It is therefore surprising that the nonequilibrium dynamics reveals the collective phenomena that we discuss in this section.

\section{A. Clustering and magnetized oscillating states}

Close to the ground state $U=-0.5|\epsilon|$, at very small temperature, an initially uniform state in $\theta$ evolves towards a two-cluster collective structure. In Fig. 16 we report $n(\theta, t)$, which shows two peaks at the cluster angles; over time the two clusters remain separated by an angle $\pi$, which makes the order parameter $\mathbf{M}=\mathbf{0}$. A negligible fraction of particles is again visiting all the circle, as for the ferromagnetic subcritical case. However, the two clusters drift on the circle, as it is shown in Fig. 16, and also by the time evolution of

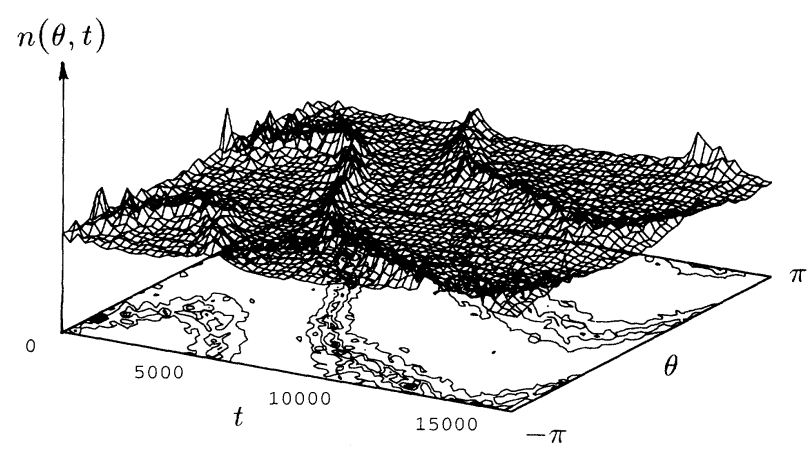

FIG. 16. Plot of the distribution function $n(\theta, t)$ for the antiferromagnetic HMF. $U \approx-0.5, N=10000$. The contour plot is also drawn in the $(t, \theta)$ plane.

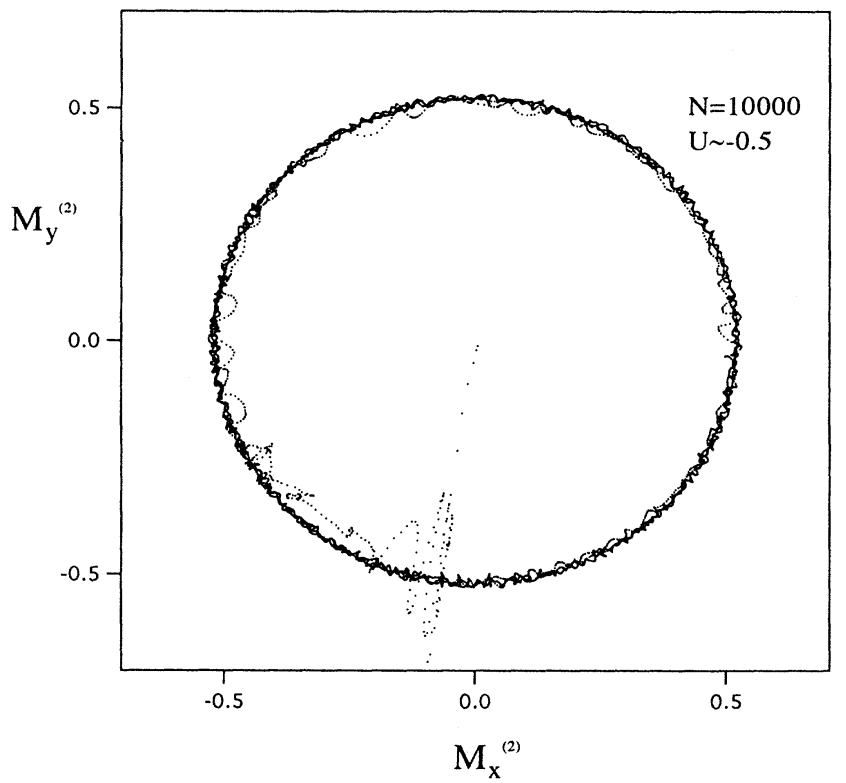

FIG. 17. $M_{y}^{(2)}$ vs $M_{x}^{(2)}$ for $U \approx-0.5$ and $N=10000$. The experiment is the same as in Fig. 16. The transient is also shown.

$\mathbf{M}^{(2)}=\left(M_{x}^{(2)}, M_{y}^{(2)}\right)=\left(\frac{1}{N} \sum_{i=1}^{N} \cos 2 \theta_{i}, \frac{1}{N} \sum_{i=1}^{N} \sin 2 \theta_{i}\right)$.

The two symmetric clusters give a coherent contribution to $\mathbf{M}^{(2)}$, producing a circular shape in the $\left(M_{x}^{(2)}, M_{y}^{(2)}\right)$ plane; see Fig. 17. The modulus of $\mathbf{M}^{(2)}$ depends on $U$ and characterizes the size of the cluster and the number of particles per cluster. The $U$ dependence of $M^{(2)}$ is shown in Fig. 18 for two different values of $N$. The curve shrinks to smaller energies as $N$ increases, revealing the possible presence of a zero-temperature phase transition, characterized by the order parameter $\mathbf{M}^{(2)}$. When kinetic energy is large enough the two-cluster state does

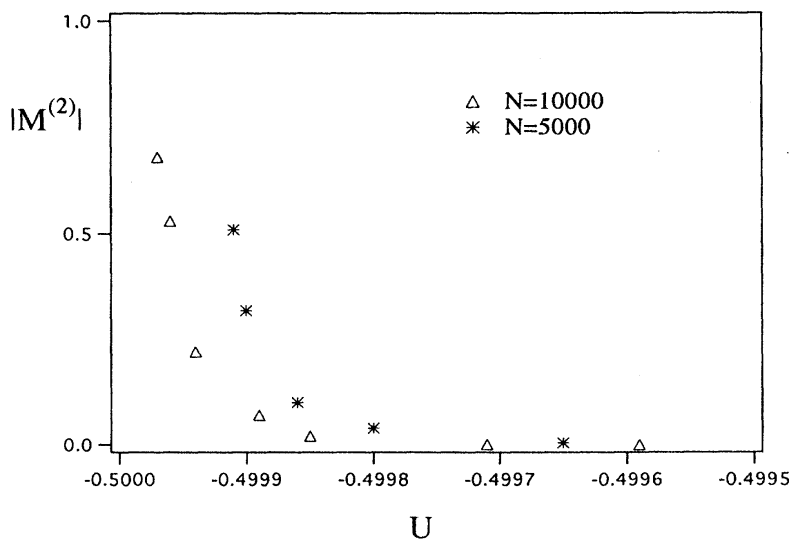

FIG. 18. Modulus of $\mathbf{M}^{(2)}$ in formula (46) as a function of the energy $U=H / N$, for $N=5000$ and $N=10000$. 


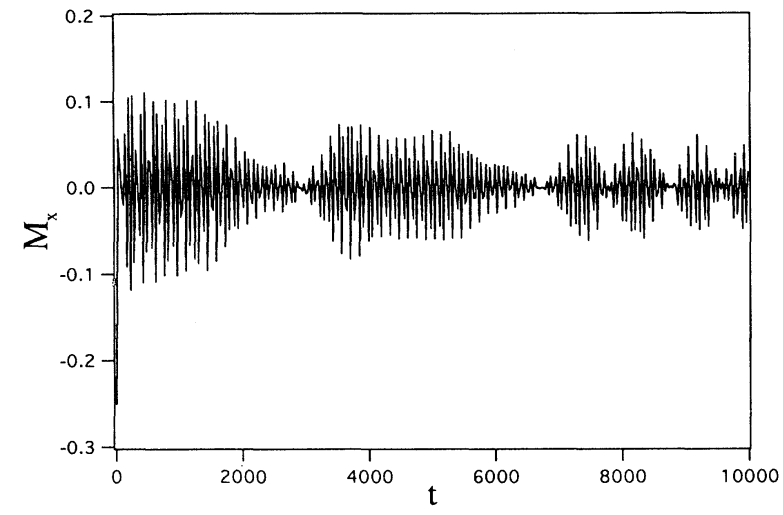

FIG. 19. $M_{x}$ vs time for the antiferromagnetic HMF. Initially we modulate $50 \%$ of the uniform spatial state with wavelength $2 \pi . U=-0.4, N=10000$. A similar, but approximately out of phase, behavior is observed for the $M_{y}$ component.

not form and the spatial distribution remains uniform.

However, it is interesting to remark that long living collective oscillations, with a sizeable amplitude, are observed when we initially modulate the spatial distribution $n(\theta, 0)$ of the particles with a wavelength $2 \pi$ even for larger energies provided $T /|\epsilon|<1$. Each component of the magnetization $\mathbf{M}$ oscillates with a non-negligible amplitude as shown in Fig. 19. Also in this case the twocluster state forms spontaneously after a short time. The maximal value of the amplitude of $\mathbf{M}$ depends on the amplitude of the initial modulation. The superposition of the drift motion of the clusters and of their vibrational motion with respect to the center of mass produces the beatings observed in Fig. 19.

It is curious to observe such a behavior since it corresponds to a long-living magnetized state, which is not expected on the basis of the equilibrium theory for the antiferromagnetic HMF. Our numerical experiences sug- gest that the damping of this state may require an exponentially long time in $N$.

\section{B. Relaxation time and high energy frequencies}

We study in this subsection the time evolution of $K_{\mathrm{SPO}}(t)$, see Eq. $(23)$, in the high energy regime for the antiferromagnetic HMF. The experimental conditions are the same as those already described in Sec. III C. We represent in Fig. 20 the relaxation time $\tau_{\text {af }}(\sigma)$ to the value $\sigma=1 / 4$ of $K_{\mathrm{SPO}}(t)$ as a function of $N T^{3 / 2}$. This law is empirically derived from numerical experiments and is again inspired by the analogy with CSM. The $N$ dependence is the same as for CSM, but the $T$ dependence is different. The relaxation is faster with respect to the ferromagnetic HMF. This difference may be the signature of the presence of some small clusters in the supercritical regime of the ferromagnetic system.

The temporal spectrum of $\mathbf{M}$ reveals a leading component as described in Sec. IIID. This is the analogous of the Bohm-Gross frequency $\omega_{\mathrm{BG}}$ observed in CSM; quantitatively it is given in formula (45), changing the sign of $\epsilon$. In Fig. 15 we show the main frequency present in the power spectrum of $\mathbf{M}(t)$ as a function of $U$. The agreement with theoretical predictions is excellent.

\section{ANALOGIES WITH SHEETS DYNAMICS}

The linear time scale of all the models introduced in this paper can be obtained by Taylor expanding the force in Eqs. (3) and (7). This establishes the analogy among $\omega_{\mathrm{HMF}}=\sqrt{|\epsilon|}$, the CSM "plasma" frequency $\omega_{\mathrm{pl}}$, and the GSM Jeans' frequency $\omega_{J}$ reported in the first line of Table I. Temperature is given by the averaged squared momentum $v_{t}$ and energy is conserved; the only subtlety concerns physical dimensions, since for the HMF

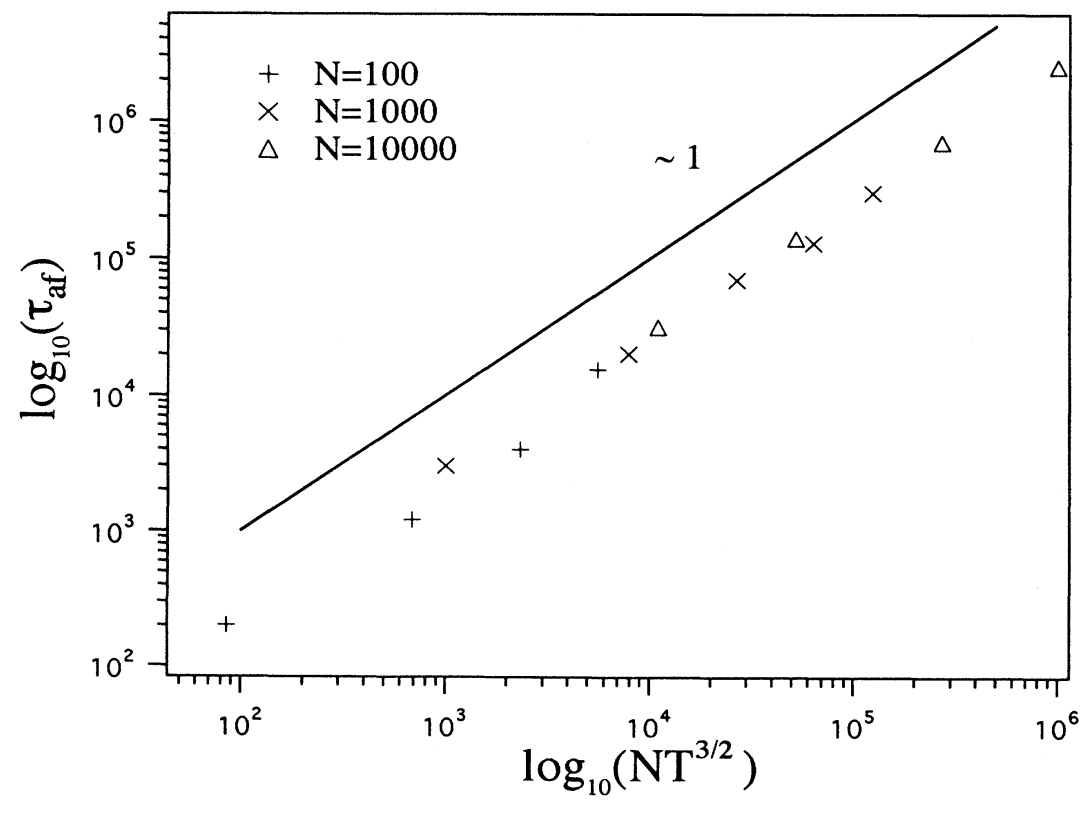

FIG. 20. Relaxation time $\tau_{a f}$ as a function of $N T^{3 / 2}$. The points are well fitted by a slope 1 . 
$\theta$ is dimensionless, so to obtain a temperature one has to multiply $v_{t}$ by the unit of length, the radius of the circle $R=1$. Through temperature and frequency one can obtain a typical length, which is the usual Debye screening length for the CSM, the Jeans' length for the GSM, and the "screening" HMF length, which characterizes the onset of collective phenomena (see line 3 in Table I). In plasmas, two different physical situations occur depending on whether we consider a length scale $\lambda$ greater or smaller than $\lambda_{D}$. If $\lambda<\lambda_{D}$, the dynamics of the particles is dominated by the short range interactions and microscopic effects are leading. No collective structures can be observed because of disorder. When $\lambda \gg \lambda_{D}$, on the other hand, collective structures appear (e.g., Langmuir modes) $[18,20]$. Disorder and individual particle effects can then be neglected with respect to large scale behavior. Following the same scheme in the GSM, the typical length corresponds to the Jeans' length $\lambda_{J}$. Hence, when $\lambda_{J}$ is smaller than the considered length, the dynamics is again dominated by macroscopic effects. There remains, however, a main qualitative difference between the gravitational and the charged sheets model: in the first model an instability leads to a collapse of the particles, while this is not the case for the second model because the interaction is repulsive. In model (1), the potential contains only one wavelength. The collective behavior thus disappears when $\lambda_{\mathrm{HMF}}>1$. This corresponds well to the presence of a phase transition in the ferromagnetic $\mathrm{HMF}$, while for the antiferromagnetic case only when $T /|\epsilon|<1$ are collective effects present if initially excited (although at very small temperature a clustering state spontaneously appears).

The grain parameter should control the typical relaxation time to equilibrium also in the HMF, as for CSM and GSM. However, although we find an agreement as far as the $N$ dependence is concerned, the time scale $\propto g_{\mathrm{HMF}}^{-1}$ (see Table I) does not have the same dependence on the temperature. Moreover, the ferromagnetic and the an- tiferromagnetic model show a different scaling. The $N$ dependence of the relaxation time implies the relevance of nonequilibrium states in the thermodynamic limit.

We are just beginning to understand the analogies and differences between magnetic system dynamics and sheet dynamics. The first natural step to go on with this study is to add a second Fourier mode to model (1) (see Fig. 2); we expect in this case a richer phenomenology and the presence of further phase transitions. We believe that this study establishes a fruitful exchange of ideas and methods between two very different fields, and that it may lead to progress in the understanding of the dynamics of systems with many degrees of freedom.

\section{ACKNOWLEDGMENTS}

We acknowledge useful discussions after the many seminars where these results were presented with F. Bagnoli, J. Bellissard, A. Crisanti, J. de Luca, E. Domany, B. Doucot, S. Fauve, H. Kantz, H. Kunz, A. Lichtenberg, R. Lima, R. Livi, R.S. MacKay, A. Maritan, H. Posch, V. Tsytovich, and A. Verga. S.R. thanks D. Escande for a very important seminal discussion which led to the study of this model. Special thanks goes to Y. Elskens for suggesting the use of the quantity (46) to characterize the antiferromagnetic case and for a very careful reading of the manuscript. M.A. thanks D. Bambusi for discussions about the drift phenomenon of the ferromagnetic cluster. M.A. acknowledges COTRAO and EC Contract No. ERBCHBGCT930295 for financial support, and the Dipartimento di Energetica dell' Università di Firenze for hospitality. He also thanks the Institute of Scientific Interchange in Torino, Italy and its Director Mario Rasetti for hospitality. Both M.A. and S.R. thank the Laboratoire de Physique Quantique in Toulouse for hospitality and S.R. thanks CNRS for financial support. This work is also part of Contract No. ERBCHRXCT 940460.
[1] S. Ruffo, Hamiltonian Dynamics and Phase Transitions, Marseille Conference on Chaos, Transport and Plasma Physics, edited by S. Benkadda et al. (World Scientific, Singapore, 1994).

[2] W. Thirring, Found. Phys. 20, 1103 (1990).

[3] O. G. Eldridge and M. R. Feix, Phys. Fluids 5, 1076 (1962); F. Hohl and M. R. Feix, Astrophys. J. 147, 1164 (1967).

[4] J. M. Dawson, Phys. Fluids 5, 445 (1962); 7, 419 (1964); Rev. Mod. Phys. 55, 403 (1983).

[5] C. J. Reidl, Jr. and B. N. Miller, Astrophys. J. 318, 248 (1987); C. J. Reidl, Jr. and B. N. Miller, Phys. Rev. E 48, 4250 (1993); T. Tsuchiya, T. Konishi, and N. Gouda, ibid. 50, 2607 (1994).

[6] M. Antoni, Ph.D. thesis, Université de Marseille (1993); M. Antoni, Y. Elskens, and D. Escande, Reduction of $N$ body Dynamics to Particle-Wave Interaction in Plasmas, in Dynamics of Transport in Plasmas and for Charged Beams, edited by G. Maino and M. Ottaviani (World Scientific, Singapore, in press).

[7] S. Inagaki and T. Konishi (unpublished); S. Inagaki (un- published).

[8] K. Kaneko and T. Konishi, J. Phys. A 25, 6283 (1992).

[9] M. Creutz, Phys. Rev. Lett. 50, 1411 (1983).

[10] M. Steiner, J. Villain, and C. G. Windsor, Adv. Phys. 25, 87 (1976).

[11] F. Casagrande et al. (unpublished).

[12] J. L. Rouet, Ph.D. thesis, Université d'Orleans (1990).

[13] D. Escande, H. Kantz, R. Livi, and S. Ruffo, J. Stat. Phys. 76, 605 (1994).

[14] R. Balescu, Equilibrium and Non Equilibrium Statistical Mechanics (Wiley, New York, 1975).

[15] A. I. Neishtadt, Fiz. Plasmy 12, 992 (1986) [Sov. J. Plasma Phys. 12, 568 (1986)]; J. L. Tennyson, J. R. Cary, and D. Escande, Phys. Rev. Lett. 56, 2117 (1986); A. V. Timofeev, Zh. Eksp. Teor. Fiz. 75, 1303 (1978) [Sov. Phys. JETP 48, 656 (1978)]; Y. Elskens and D. Escande, Physica D 62, 66 (1992).

[16] M. R. Feix and F. Hohl, Expériences Numériques sur les Modèles Stellaires Unidimensionnels (Editions du CNRS, Paris, 1968), pp. 289-298.

[17] R. Balescu, Phys. Fluids 3, 52 (1960); A. Lenard, Ann. 
Phys. 3, 390 (1960).

[18] F. Chen, Plasma Physics and Controlled Fusion (Plenum, New York, 1988); D. R. Nicholson, Introduction to Plasma Theory (Wiley, New York, 1983).

[19] L. D. Landau and E. M. Lifchitz, Cinétique Physique
(Mir, Moscow, 1990).

[20] D. Bohm and E. P. Gross, Phys. Rev. 75, 1851 (1949); 75, 1864 (1949); D. Bohm and D. Pines, ibid. 82, 625 (1951); D. Pines and D. Bohm, ibid. 125, 338 (1952). 